\title{
ALL-OPTICAL NETWORKS PERFORMANCE IMPROVEMENT BY SOLVING THE RWA PROBLEM USING GENETIC ALGORITHM
}

\author{
MOHAMMED GHANIM SAEED ${ }^{1,{ }^{*} \text {,and FiRAS MAHMOOD MUSTAFA }}{ }^{* *}$ \\ *Technical Institute of Shekhan, Duhok Polytechnic University, \\ Kurdistan Region-Iraq \\ *** College of Engineering, Nawroz University and Duhok Polytechnic University, \\ Kurdistan Region-Iraq
}

(Accepted for Publication: December 8, 2020)

\begin{abstract}
The problem of significant importance in the design of all-optical networks is the Routing and Wavelength Assignment (RWA) problem that depends on the light path route and wavelength using different algorithms. The main aim of (RWA) is to maximize the number of established connections. The significant indication by applying the RWA is to diminish the resources of the network demanded to serve the same number of requests. Consequently, the total number of requests served by the same resources will be increased. The main aim of this research is to manage the RWA algorithm by inspecting the performance of each algorithm in static (Fixed Routing Algorithm and Fixed Alternate Routing Algorithm) and dynamic routing algorithms in an optical network. With this work, the comparison between various RWA algorithms is performed using sorted shortest path algorithm and non-sorted random algorithm. Extra investigation and comparison can be achieved via the hiring Genetic algorithm (GA) as a tool to enhance optical network performance. The proposed techniques are applied to the design of some topologies depending on the number of nodes and the set of connecting links. With this work, it is assumed that all nodes are with no wavelength conversion and the identical load was utilized on the network by using three different algorithms. The comparison between the three algorithms shows that the dynamic algorithm gives good throughput and less resource usage. Besides, it has been shown that the shortest path algorithm yields results better than a random path algorithm. Finally, by using the GA tool in the optimization, the results have been obtained within the accepted rate.
\end{abstract}

KEYWORDS: All-optical networks; Routing and Wavelength Assignment (RWA); Genetic algorithm; sorted shortest path algorithm; Network performance optimization.

\section{INTRODUCTION}

$\mathbf{T}$ he Internet is a global network connecting a huge number of devices, a large number of countries around the world are linked into exchanges of data, and they can send and receive all kinds of information such as text, graphics, voice, video, news, opinions, and computer programs. The increasing growth of Internet traffic has put a significant demand for Internet capacity (Melanie M., 1999). So, the network needs to serve the growth of internet traffic for accepting a larger number of requests and decrease the blocking requests by using minimal resources as shown in figure 1. An optical network provides a common infrastructure over which a variety of services can be delivered. These networks are also increasingly becoming capable of delivering bandwidth flexibly where and when it is needed. Optical fibers offer much higher bandwidth than copper cables and other undesirable effects. The problem of routing and wavelength assignment plays the main role in the optical fiber to achieve the high number of requests to be accepted and served with minimizing the congestion traffic. Methods proposed to solve the RWA problem can be used as two operations; the first one which is called the routing is by finding all possible paths from source to the destination node. Some of the routing algorithms are listed below (Wason A., 2011):

- Fixed routing: The path for each source-destination pair is calculated off-line using an algorithm. For example, the Dijkstra Algorithm.

- Fixed alternate routing: Instead of calculating one path for each pair, fixed alternate routing 
calculates off-line two paths for each pair.

- Adaptive routing: The paths are calculated on-line, depending on the network state, which reflects the resource usage.

The second operation of the RWA problem is called the wavelength assignment operation, which aims to find any available wavelength within each fiber to carry the requested bandwidth of the demand. Both routing and wavelength assignments can be formulated as graph-theoretic problems (Rashed A. et al, 2011). One of the important resources that must be minimized is the time. Therefore, the process of accepting requests at the shortest possible time working to increase the chance of accepting other requests in the same network. There are usually two types of methods to solve the RWA problem.

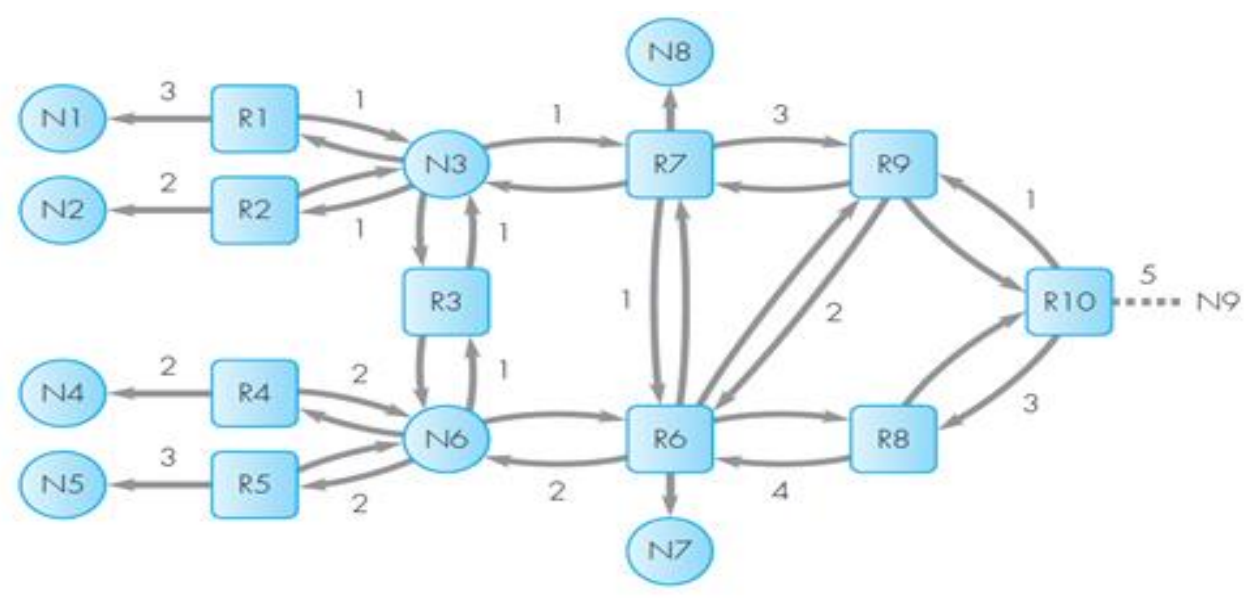

Fig. (1): Routing in network (Saeed M. G., 2013).

The first type can be solved through linear programming (LP) and the second type can be solved through a heuristic algorithm. However, in optical networks with high dynamic topology structure and time delay constraint, the computational complexity of the LP method increases sharply with the expansion of network size, so this kind of method is not suitable for the solution of the RWA problem of the large-scale optical network (Sun 2019). There are two types of nodes in fiber optic networks:

The first type contains wavelength conversion: this type is more complex, expensive, and needs more resources including the time.

- The second type is without wavelength conversion: this type is more flexible than the first type but needs to apply the wavelength continuity constraint, which assumes that the same wavelength must be used in all links through the route between the source node and the destination node.

In this work, all nodes are without conversion, and the Genetic Algorithm (GA) is used to optimize the number of accepted requests by finding a suitable number of wavelengths for each topology in the shortest possible time and using less resource as possible. The GA tries to solve the RWA problem to provide a group of optimal or sub-optimal routing scheme.

\section{RELATED WORKS}

In 2011, Rashedi A. and others, (Rashed A. et al, 2011) Proposed an Intelligent System to apply an Artificial Bee Colony (ABC) Algorithm to solve Dynamic RWA problem by minimizing the Blocking probability, the simulation result demonstrates that the algorithm $\mathrm{ABC}$ based DRWA algorithm has better performance in reducing the Blocking probability comparing to the Fixed-alternate routing algorithm. In 2013, Chatterjee B. Ch. and others (Chattenjee B.Ch. et al, 2013) Discussed different conventional RWA approaches in the Wavelength-Routed optical network and compare them with proposed PRWA scheme under wavelength continuity constraint, founded that the performance of First-Fit wavelength assignment mechanism in terms of (BP) Blocking Probability is better than other 
wavelength assignment scheme. In 2016, Monoyios and others, (Monoyios D. et al., 2016) proposed genetic algorithms for solving the RWA problem during the design phase of a transparent WDM optical network to minimize in-band and out-of-band crosstalk interactions, thus minimizing the spread of high-power jamming attacks in the network, while keeping the network cost low. The proposed Crosstalk-Aware GA algorithm has performance close to the corresponding ILP algorithm in terms of the number of lightpath interactions, while at the same time having performance close to the Simple GA algorithm in terms of network resources (number of wavelengths required). While Lechowicz P. and Walkowiak K., (Lechowicz P., 2016) proposed the GA-MSF algorithm for the RSA problem and provided its detailed description. We performed numerical experiments to find the best parameters and strategies set. Finally, we compared the efficiency of GA-MSF with reference algorithms: KW and MSF. Obtained results show that GA-MSF can provide good spectral utilization for scenarios with small demands' set and a big number of candidate structures. In 2017, Hsu et al., (Hsu Ch. et al, 2017) presented an algorithm for solving the RWA problem. The algorithm combines the ideas of the bin-packing method and edge-disjoint paths. It considers only several requests at a time and solves the corresponding MEDP problem. The remaining requests are then scanned in a backward manner such that the one with shorter shortest paths tries to fit into the existing wavelengths first. The proposed algorithm is capable of solving small and medium-size instances in a reasonable time. In 2018, Batista C. et al., (Batista C. et al, 2018) presented a genetic algorithm (GA) approach to solve the RWA problem in WDM networks, transparent optical networks with static traffic. Simulation results showed that the GA performed better than the standard approaches for both routing, such as fixed (Dijkstra) and fixed-alternate routing (Yen); and wavelength assignment subproblems (sequential graph coloring and first-fit) in terms of blocking probability. Yen's algorithm was able to compute link-disjoint paths, which may have improved a lot the performance of the fixed-alternate routing approach over Dijkstra's fixed routing. However, the combination of least congested path technique with general objective function (GOF) algorithm inside the working mechanism of the proposed genetic algorithm did contribute to achieving as a lightly better result. In 2019, Kaur H., and Rattan M., (Kaur H., 2019) presented an efficient solution for creating light paths using static traffic in WDM optical networks. They proposed two parallel hybrids methods for the optimization multi-objective problem. The idea of using a parallel hybrid is to incorporate and use the best of features of all three algorithms in optimizing the performance metrics of WDM networks, i.e., blocking probability, load, and several wavelengths. Assessment of blocking probability for different values of Erlang load per link concerning the number of connection requests is performed using the proposed algorithms.

\section{ROUTING AND WAVELENGTH ASSIGNMENT (RWA)}

A lightpath is a path taken by the signal from the source to destination nodes in optical form through intermediate links and it has to be established before communication between the two nodes. So, to establish a lightpath in a WDM network, it is necessary to determine the route over with the lightpath that should be established and wavelength to be used on all the links along the route. The RWA problem is significantly more difficult than the routing problem in the electronic network. The additional complexity arises from the fact that RWA is subject to the wavelength continuity constraint and distinct wavelength constraint (Rouskas G.N., 2002). The routing is the first part of the RWA problem, and it is the selection of a suitable path (lightpath) for each connection request between the source and destination (s-d) nodes in the network. Many of the routing technique used for an optical network are borrowed from methods used for traditional circuit-switched telephone networks (Mokhtar A., 1998).

The wavelength assignment is the second part of the RWA problem. This part is required to select a wavelength for a given lightpath if there are multiple feasible wavelengths between the source and destination nodes. The wavelength selection may be performed either in parallel with a route-finding or after a route has been determined (in this research we assume wavelength assignment occurs after the route-finding (Jue J.P., 2001).

The Optical Cross-Connects (OXC's) are used to route wavelengths between input and output ports while adding, and dropping the local traffic. 
The main function of the $\mathrm{OXC}$ is to dynamically reconfigure the network at the wavelength level for restoration or to accommodate changes in bandwidth demand. OXC systems are expected to be the cornerstone of the photonic layer providing carriers more dynamic and flexible options in building network topologies with enhanced survivability (Kaya, Y. et al, 2011). The router that performs routing based only on the label is called a label switch router (LSR) or transit router. This is a type of router located in the middle of the network. It is responsible for switching the labels used to route packets. The RWA problem in an optical network is illustrated in figure 2, where it is assumed that each fiber support three wavelengths, the effect of the wavelength continuity constraint is represented by replicating the network into as many copies as the number of wavelengths (three in this case). Thus, finding a path for a connection may potentially involve solving $\mathrm{W}$ routing problems for a network with $\mathrm{W}$ wavelength, one for each copy of the networks. The wavelength continuity constraint may be relaxed if the nodes are equipped with wavelength converters (Rouskas G.N., 2002), in this research; it is assumed that no wavelength conversion is available in the network nodes. Whenever a call arrives at a wavelength router, it will run a predefined algorithm and select the outgoing port and a wavelength. If a route with the capacity of one lightpath between the two nodes cannot be found (there is no available wavelength in that route), the lightpath is rejected (blocked) and the request cannot be granted, otherwise, the lightpath is allocated (Glenstrup A. J., 2002).

The selection of the wavelength plays an important role in the performance of the algorithm and also on the overall blocking probability. Hence a wavelength router (WR) has to find the router for the lightpath and assign wavelength that minimizes the blocking probability. This function is for fundamental importance in the design of all-optical networks (Fenger C., 2004). The RWA problem can be solved either in one phase, where both route and wavelength are assigned at the same time or in two phases where first the route is selected and then a feasible wavelength assignment is determined for the given route. The traditional way to solve the RAW problem is to determine the routes for all connections first and then assign wavelengths to connections (Ozdaglar A. E., 2003).

The RWA problem can be considered under two different traffic assumptions, the static (off-line) and dynamic (on-line) traffic RWA problem (Mustafa F. M., 2017). The static traffic RWA applies to the case in which the set of connections is known in advance i.e., all the lightpaths to be set up in the network are known beforehand. The static RWA arise naturally in the design and capacity-planning phase of architecting an optical network to determine capacity need in the future, where the objective is to accommodate the demands (lightpaths) while minimizing the number of wavelengths used on all links, alternatively one may attempt to set up as many of these connections as possible for a given number of wavelengths i.e., the object is to maximize the total throughputs in the network (Jue J.P., 2001). The static RWA problem can be formulated as an integer linear program (LIP), the objective is to minimize the number of wavelengths required to establish all of the given connection requests or to maximize the number of connection that is routed with the existence of a constraint on the number of wavelengths. The following notation is used (Lea D. P., 2004).

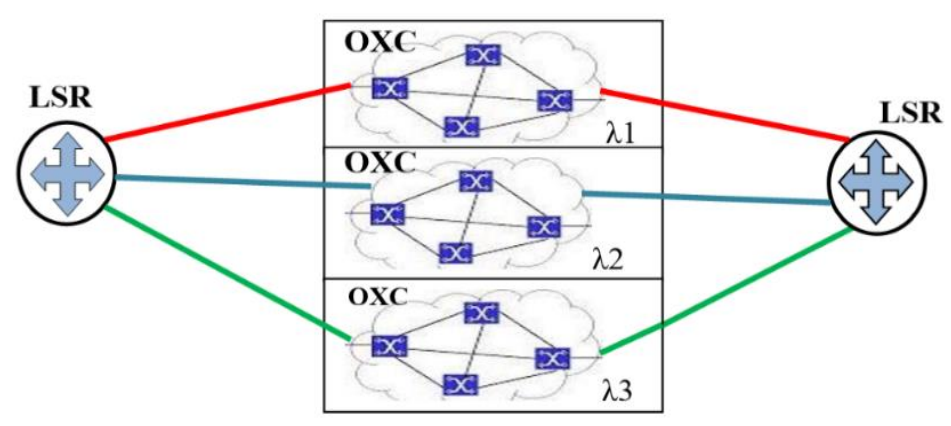

Fig. (2): The RWA problem in optical networks, with three wavelengths per link (Saeed M. G., 2013).

mohammed.saeed@dpu.edu.krd; firas.alfaqe@nawroz.edu.krd

${ }^{1}$ Corresponding author: College of Engineering, Nawroz University, Kurdistan Region, Iraq 
- $\mathrm{J}$ is the number of source-destination ( $\mathrm{s}, \mathrm{d}$ ) pairs in the network.

- $\mathrm{L}$ is the number of physical links.

- W is the number of wavelengths available on each link. It is assumed to be the same for each link.

- $\rho \mathrm{j}$ is the offered load between ( $\mathrm{s}, \mathrm{d}$ ) pair $\mathrm{j}$ for $\mathrm{j}=1, \cdots, J$.

- $\mathrm{P}$ is the total number of available paths that can be used to route connections.

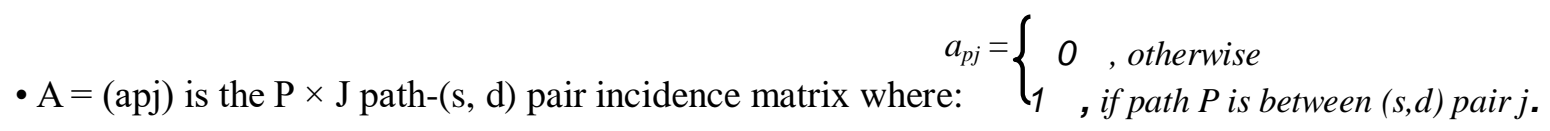
$\cdot \mathrm{B}=(\mathrm{bpl})$ is the $\mathrm{P} \times \mathrm{L}$ path-link incidence matrix where $\div=\left\{\begin{array}{ll}1 & \text {, if link lis on path } p, \\ 0, & \text {, otherwise }\end{array}\right.$ Define variables:

- $\mathrm{mj}$ as the number of connections carried between (s, d) pair $\mathrm{j}, \quad$ for $j=1, \cdots, J$.

- $\mathrm{C}=(\mathrm{Cpw})$ as the $\mathrm{P} \times \mathrm{W}$ path-wavelength assignment matrix where:

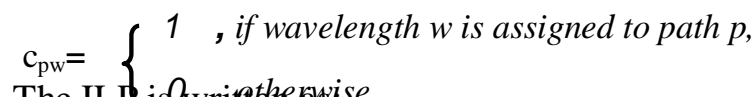

The ILP is Owritethregisise

$$
\text { Maximize } \sum_{j=1}^{J} m j
$$

Subject to:

$$
\sum_{p=1}^{p} \mathrm{bp} 1 \mathrm{cpw} \leq 1, \text { for } \mathrm{w}=1, \cdots, \mathrm{W}, \text { and } \mathrm{l}=1, \cdots, \mathrm{L}
$$

$$
\mathrm{mj} \leq \sum_{w=1}^{w} \sum_{p=1}^{p} \text { apjcpw }, \text { for } \mathrm{j}=1, \ldots, \mathrm{J}
$$

$\mathrm{mj} \leq \rho \mathrm{j}$, for $\mathrm{j}=1, \cdots, \mathrm{J}$

$\mathrm{mj} \geq 0$, ms: integer, for $\mathrm{j}=1, \cdots, \mathrm{J}$

$\mathrm{cpw}=0$ or 1 , for $\mathrm{p}=1, \cdots, \mathrm{P}, \mathrm{w}=1, \cdots, \mathrm{W}$

Equation 2, provide the constraint that ensures the same wavelength is used at most once on each link, while equation 3 , provide the constraint that represents a bound on the amount of carried traffic for (s, d) pair $\mathrm{j}$, and equation 4 , provide the constraint that ensures the amount of traffic carried by the network is no more than the offered load. The total number of variables for this formulation is $\mathrm{P} \times \mathrm{W}+\mathrm{J}$. Given that the number of paths $\mathrm{P}$ can increase exponentially with the number of network nodes and links, the RWA problem is usually addressed by using heuristic techniques (Lea D. P., 2004).

In the dynamic RWA, calls arrive at the network node dynamically and remain for some time (random holding time) before departing, dynamic routing plays a major role in the reliable operation of the large-scale network (Chen, L., 2005). Instead of minimizing resource as in the static traffic case, the object in the dynamic situation is to choose a route and wavelength that maximize the probability of setting up a given connection, while at the same time attempting to minimize the blocking probability (Chu, X. et al., 2003). Blocking probabilities in the dynamic traffic RWA exist in a scenario where the capacities in a network have been decided and dynamic traffic demands, known as calls arrive. Call arrivals can be modeled as stochastic processes, the most basic arrival process used to characterize arrivals in a telecommunication network is the Poisson 
process which is stationary, independent, and regular. The interarrival time $t$ of the Poisson process follows a negative exponential distribution (Glenstrup A. J., 2002), as shown below:

$$
F(t)=1-e^{-\lambda t}
$$

Where: $\lambda$ is the arrival intensity indicating the average number of the arrivals per time unit.

The Poisson process is the best for modeling call arrivals from a large set of sources that are not expected to be correlated (e.g., telephone subscribers). A multitude of other distributions have been devised for modeling other scenarios like overflow traffic, traffic from a limited set of sources .etc., each call will be associated withholding time, which is the time that the call will occupy the connection when it is served (Glenstrup A. J., 2002). Where any blocked calls disappear there is no mechanism set up for retransmitting the call and do not affect the future behavior of the system. The source of call blocking can be one of the following (Hsieh T., 2003):

1- A free wavelength cannot be found on all links of the request path; the wavelength continuity cannot be satisfied.

2- One of the links on the route full; all of the wavelengths have already been allocated to other connection requests.

\section{GENETIC ALGORITHMS (GA)}

The genetic algorithms are investigation algorithms that depend on the appropriate, survival supposition of Darwin (Melanie M., 1999). A set of proper solutions are provided (population) for the genetic algorithm (GA). The solution is encoded for a concatenation (chromosome) and tested with some fitness computation. The real solution to be tested is the phenotype and the encoded solution is known as the genotype. The GA generates new solutions (children) at each restoration by applying the genetic processes on the chosen solutions (parents). Fitness estimate of the existing solutions is permanently performed and fitness solutions are chosen for the next restoration as a new population. Specific optimization issues, such as scheduling, workshop schedule, and games can be solved by GAs (Sivanandam S.N, 2008).

\subsection{Standard Genetic Algorithm}

World John Holland was able to put the basic rules of GA based on his study of fruit flies society as well as it called (simple genetic algorithm). After selecting the issue to be resolved and the representation of solutions with a series of bits, then the basic steps for most applications genetic algorithm can be summed up as shown below (Melanie M., 1999):

1- Start creating a random population, which means (l) bits are the solutions to the question that are presented, to compare the size of the population to (n) the people.

2 - Find the fitness $f(x)$ of the population of each individual

3- Repeat the steps before gene (n) is composed:

Choosing the pairs of current parents and their chances of being selected are increasingly dependent on the degree of quality- then selecting occur (With replacement), this means selecting a parent several times.

Probably the $\mathrm{cm}$ (crossover probability) or (crossover rate) formed two genes when the crossing did not only take place in the manufacture of a replica of parents. The GA will apply another crossover; this reflects other points that don't like early crossing genes. Adjust the Pm (Mutation Probability) or (Mutation Rate) of two genes at a similar location and introduce current individuals to a new population.

4- Modification of existing demographics with new populations.

5- Return to stage 2.

The ideal generation is called every iteration of these steps and consistency depends on the process.

\subsection{Main Features of Genetic Algorithms 4.2.1 Genetic Algorithm Encoding:}

Encoding is an individual gene representation method. This can be done with bits, numbers, trees, arrays, lists, or any other object. The encoding primarily depends on the solution. Binary strings and other forms like Octal, Hexadecimal, Permutation, Value, and Tree are the typical way of encoding (Sivanandam S.N, 2008). This is the binary encoding:

Chromosome 1: 11011010 1:00 11:011

$$
1100
$$

\subsubsection{Fitness Function in Genetic Algorithm}

Fitness Function is a key component of the desired problem solution (i.e. cheapest price, shorter route, most compact arrangement, etc.). This function determines the fitness of a particular solution and returns it. As shown below, the fitness function:

\footnotetext{
mohammed.saeed@dpu.edu.krd; firas.alfaqe@nawroz.edu.krd

${ }^{1}$ Corresponding author: College of Engineering, Nawroz University, Kurdistan Region, Iraq
} 


\section{$F(x)=1 / f(x)$, where: $f(x)$ is the objective function.

All solutions with "poor" fitness are discarded and approved by the rest of the genetic algorithm. "good" interest of fitness. A fitness function aims to give the genes a meaningful, measurable, and comparable value (Yussof S. et al, 2009).

\subsubsection{Selection in Genetic Algorithm}

The selection operator defines the way people are selected for reproduction in the current population. There are many techniques (e.g. roulette, party, match, Boltzmann, random selection, etc.), but typically the more appropriate people are chosen (Sivanandam S.N. 2008). There are many strategies.

\subsubsection{Crossover in Genetic Algorithm}

Overlap the genetic operator who brings together (mates) two chromosomes, to produce a new (offspring) chromosome. The theory behind crossover is that if each parent chooses the best traits, the new allele will be different for both parents. A crossover takes place via user-definable through development. Odds (e.g. one point, two-point, sometimes crossover, etc.). Crossover likelihood. This director applies heritage properties (their ancestors' descendant ancestry genes) (Kaya, Y. et al, 2011).

\subsubsection{Mutation in Genetic Algorithm}

The mutation operator creates spontaneous variations of the off-spring genetic codes. This operator is needed in the genetic code to incorporate some random diversity. In some cases, without a mutation operator (local maximum problem) GA can find the optimal solution. Besides, mutation stops the GA from collapsing into local extremes. Mutation does not happen too much, because then GA transforms into a random quest.

-Flip Bit Mutation

-Boundary Mutation

-Non-Uniform Mutation

4.3 The Genetic Algorithm Tool

The GA Tool provides the possibility to use the genetic algorithm without the command line operation. To open the GA utility in the Matlab command prompt, enter (Gatool) and the program opens. The following information must first be entered in order to use the GA tool (Saeed M. G., 2013):

The fitness function in @fitnessfun format, where fitnessfun.m is an M-file to calculate the fitness function. "Write an M-File to optimize your work."

-Number of variables - the fitness function input vector length. To execute the GE algorithm, click the Start button. The tool displays the results of the optimization in the Status and Results panel.

\section{ROUTING AND WAVELENGTH ASSIGNMENT SIMULATION}

A system that is designed in this proposal to solve the (RWA) problem through the use of Matlab tool for this design, the GA is also used to improve results and to increase the number of accepted requests received in the network by Minimize the declined requests by finding the best way to do so while at the same time trying to reduce the resources used as much as possible by selecting the correct number of wavelengths at the shortest possible time. Matlab language suits the use of such algorithms as a simulation. Different topologies have been used to ensure a sufficient number of results and appropriate results in contrast and to apply the same topology to more than one algorithm when the program runs. The first step is the assumption of a specific topology depending on the number of nodes and the complete links to the designed system and the topologies used in the design for testing the performance of various algorithms, illustrated in figure 3 , with the Network links for topology;

and a variety of matrices. The type of requests can be calculated using equation 9 :

$$
\mathrm{K}=(\mathrm{n} * \mathrm{n}-1) \quad \text { where: } \mathrm{n}=\text { number of node in the topology }
$$




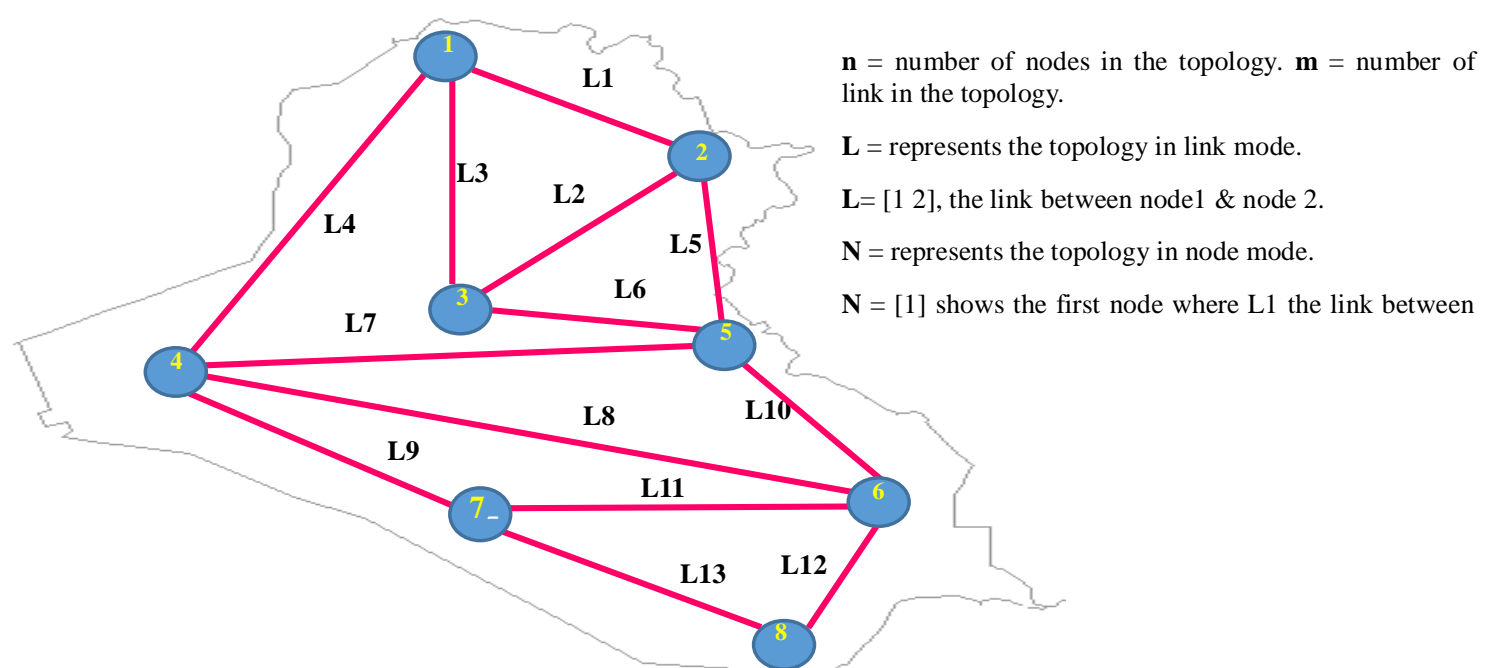

Fig. (3): The used topology (Iraq map) with 8 nodes \& 13

The types of requests can be represented for $t$ opology in figure 3 by the following matrix:

$\mathrm{K}=[(1,2),(1,3),(1,4),(1,5),(1,6),(1,7),(1,8),(2,1$ )$,(2,3),(2,4),(2,5)(2,6),(2,7),(2,8)$, $,(8,1),(8$ $, 2),(8,3),(8,4),(8,5),(8,6),(8,7)]$. For each pair in the list of the types of requests has two values, the first is the source at the left,

While the second at the right describes the destin ation.

\subsection{Sorting, Finding and choosing paths}

The shortest way (Sorting) can be found by using equation 10 below to find all possible routes among nodes in any topology Dijkstra algorithm that uses two algorithms (Shortest path \& randomly).

\section{Table B= Sortrows(Table A, mode)}

This means sort table $\mathrm{A}$ in the direction selected by mode. The shortest paths in this study concern the path requiring the minimum number of links known as the "Hop-Count routing" where each link takes into account the same length and gives a value of 1. Two-hop routes are usually the same (equal 2), and the smaller number of hop routes is the shorter route.

\subsection{Wavelengths Assignment simulation}

In order to satisfy the request from source to destination, the channel assignment restrictions should be followed, so that each topology node is of the form WSXC, which ensures that the wavelength is not transformed possible and the continuity constraint of the wavelength must be met. The wavelength conversion involves effort and difficulty during the conversion process, and for this reason, every node in the used topologies was without wavelength conversion. The random wavelength assignment technique for RWA was also used in the proposed method.

\subsection{Generating Requests and Calculate Accepted Rate}

In every iteration, the generation randomly depends on the types of requests of the network, and after that the percentage is clear, the number of iterations in each topology is 10,000 . Multiple iterations help to obtain results with high precision as requests are generated randomly, The Matlab code that is shown below in figure 4, explains generating types of requests at each iteration randomly.

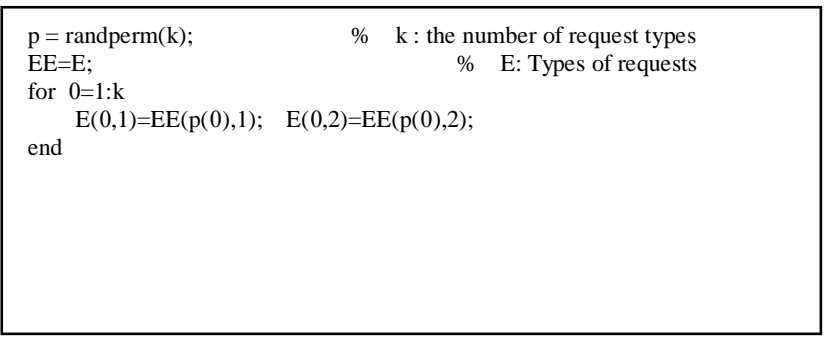

Fig. (4): Pseudo code to generate the Types of Requests Randomly.

mohammed.saeed@dpu.edu.krd; firas.alfaqe@nawroz.edu.krd

${ }^{1}$ Corresponding author: College of Engineering, Nawroz University, Kurdistan Region, Iraq 
As for wavelength, it starts with one wavelength and rises slowly over time to the saturated state (accepting as many requests as possible in the network). The saturation condition means the percentage is reached by equation 11:

$$
\text { Accepted Rate }=\frac{\text { No of accepted request }}{\text { offered load }} \%
$$

This method is applied to a fixed algorithm, as well as to alternative algorithms, using the shortest paths (with sorting) or randomly (without sorting), and also using the Dynamic Algorithm.

\subsection{Accepting Requests in Static \& Dynamic Routing Algorithms}

To accept any request from $\mathrm{S}$ to $\mathrm{D}$ in the case of wavelength, all link paths should be of the same type, except that all link paths connecting the $\mathrm{S}$ and $\mathrm{D}$ nodes should not be busy.

\subsubsection{Static Routing}

One or two routes are used in the static routing, meaning that only one or two routes are used to support the request in the network, and if they are busy, the request will be refused. The static routing is quick and easy to program, and is ideal for network design. The route depends on the number of hops among the nodes and finds the shortest path or finds the path randomly either in the case of an equal number of hops of the first kind (shortest path) through which the choice between them will be random. To accept any request obtained in any topology, certain steps should be taken as the following:

a) Fixed Routing (only one fixed path): The first step is to find all possible paths and save them in the table then based on the type of algorithm used, pick only one path from the table as in the shortest path algorithm (with sorting) selecting the shortest path of all possible paths or randomly (without sorting) selecting the first path of the list. Table 1 displays the matrices of the topology used which is the map of destination for Iraq.

Table 1. Represent matrixes of topology of Map of Iraq.

\begin{tabular}{ccccccccc}
\hline \multicolumn{1}{c}{ a) } & \multicolumn{7}{c}{ E-matrix for Iraq topology. } \\
\hline $\begin{array}{c}\text { Node } \\
\mathbf{s}\end{array}$ & 1 & 2 & 3 & 4 & 5 & 6 & 7 & 8 \\
\hline $\mathbf{1}$ & 0 & 1 & 3 & 4 & 0 & 0 & 0 & 0 \\
\hline $\mathbf{2}$ & 1 & 0 & 2 & 0 & 5 & 0 & 0 & 0 \\
\hline $\mathbf{3}$ & 3 & 2 & 0 & 0 & 6 & 0 & 0 & 0 \\
\hline $\mathbf{4}$ & 4 & 0 & 0 & 0 & 7 & 8 & 9 & 0 \\
\hline 5 & 0 & 5 & 6 & 7 & 0 & 1 & 0 & 0 \\
\hline 6 & 0 & 0 & 0 & 8 & 10 & 0 & 11 & 12 \\
\hline 7 & 0 & 0 & 0 & 9 & 0 & 11 & 0 & 13 \\
\hline 8 & 0 & 0 & 0 & 0 & 0 & 1 & 13 & 0 \\
\hline & & & & & & 2 & & \\
\hline
\end{tabular}

\begin{tabular}{cccccc}
\hline \multicolumn{7}{c}{ b) L-Matrix } \\
\hline L1 & 1 & 2 & L8 & 4 & 6 \\
\hline L2 & 2 & 3 & L9 & 4 & 7 \\
\hline L3 & 3 & 1 & L10 & 5 & 6 \\
\hline L4 & 4 & 1 & L11 & 6 & 7 \\
\hline L5 & 5 & 2 & L12 & 6 & 8 \\
\hline L6 & 3 & 5 & L13 & 7 & 8 \\
\hline L7 & 4 & 5 & & & \\
\hline
\end{tabular}

\begin{tabular}{ccccc}
\hline \multicolumn{5}{c}{ c) N-Matrix. } \\
\hline N1 & 1 & 3 & 4 & 0 \\
\hline N2 & 1 & 2 & 5 & 0 \\
\hline N3 & 3 & 2 & 6 & 0 \\
\hline N4 & 4 & 7 & 8 & 9 \\
\hline N5 & 5 & 6 & 7 & 10 \\
\hline N6 & 10 & 8 & 11 & 12 \\
\hline N7 & 9 & 11 & 13 & 0 \\
\hline N8 & 12 & 13 & 0 & 0 \\
\hline
\end{tabular}

b) If the alternative algorithm is used, then two paths are used when using one path with a fixed algorithm. Identify and store all possible paths between the two nodes in a special table according to the algorithm used to find the correct paths if the first path did not meet the request.

\subsubsection{Dynamic Routing}

The same method as in the fixed path or alternative paths but it just varies in that the route finding process will take place after receiving the order. All demands are met and departed sequentially at random. Whereas in a
Static Algorithm the method of finding a direction will be in advance. Thus all available paths are used to serve any request received in the network instead of using one or two paths. To accept the request in the Dynamic algorithm, all available paths should be used to satisfy the request unless the available paths are busy in this case. The chosen path is used to satisfy the request if the algorithm fails to choose another path, and so on, according to the algorithm used in finding the paths. But, if all the chosen paths did not meet the request, the request would be denied. 


\subsection{Genetic Algorithm}

Repeat previous static and dynamic routing operations using a genetic algorithm feature, using previous system design to work with the genetic algorithm on MATLAB. By opening the Matlab window calling the genetic function gatool at the command window, the genetic window will appear containing certain parameters that only need to be changed with the system design depending on the topology configuration and the number of wavelengths needed, where the higher number of wavelengths will increase the number of variables set. Integer can't have decimal places as the number of wavelengths is incremented one by one so the form of declaration is BitString type. There is a big part and a secondary part of the parameters in the genetic algorithm tool, in this research only a big part needed. The genetic algorithm will minimize the objective function to give an ideal solution for our problem definition. Initial individuals for the genetic algorithm population will be generated using the creation function.

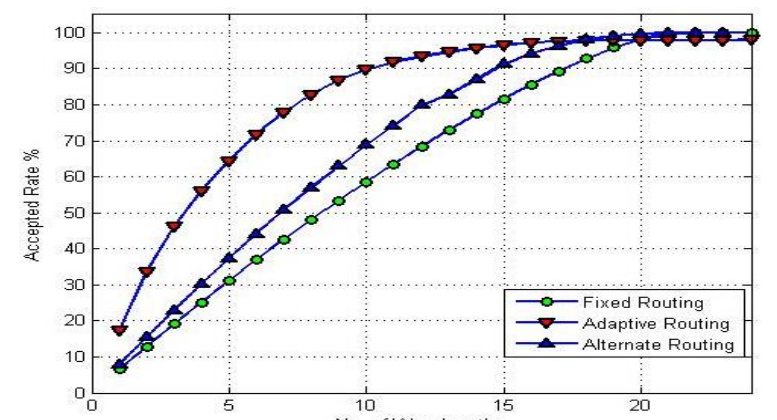

Figure 5: Three routing af Wolengths

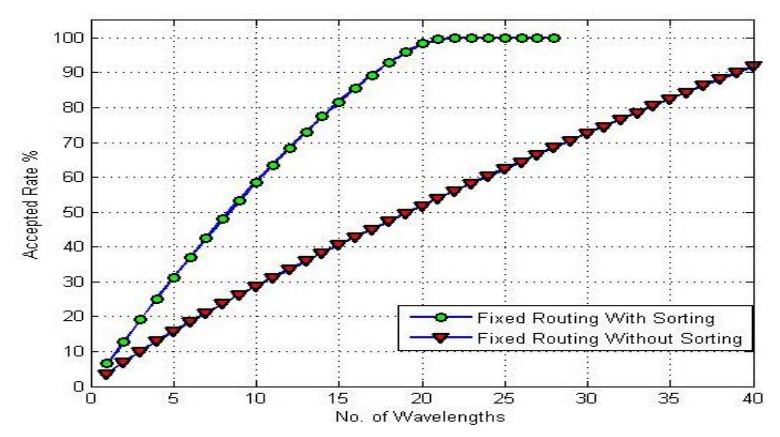

Fig. (7): Comparison fixed algorithm with and without sorting.

The results presented by the figures 5-8 shows that by applying the methods on the selected topology, the adaptive routing algorithm is better than the fixed and alternate routing algorithms. The fixed routing algorithm has less allowed accepted rate as compared with the alternate and adaptive routing algorithms, and it
Writing the program in Matlab language and saving as an $\mathrm{M}$ file to call the objective function (Static and Dynamic Routing) then running the GA program until it stops when finding the property (wavelength) as the binary number.

\subsection{RWA Algorithms Test}

Initially, a topology was chosen and checked with a single wavelength with three routing algorithms (fixed, alternative, and adaptive) with sorting after that by increases the number of wavelengths by one to demonstrate the behavior and efficiency of the network in-wavelength range.

\subsection{Simulation Results and Discussions}

Figure 5 shows the result of each routing algorithm with the sorting step, while figure 6 shows the result of each routing algorithm without the sorting step. The $\mathrm{x}$-axis indicates the number of wavelengths has been used at each point, while the y-axis indicates the accepted rate ratio that reaches 100 percent (Saturation state).
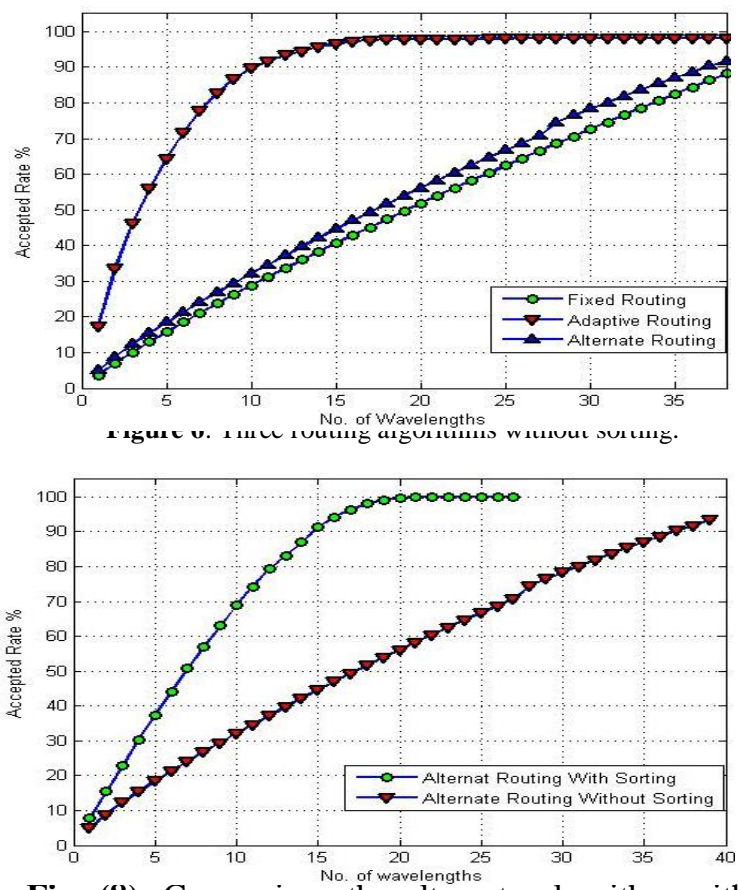

Fig. (8): Comparison the alternate algorithm with and without sorting.

consumes less time than other algorithms. The alternate routing algorithm has a better result than the fixed algorithm and less than an adaptive routing algorithm with consumes less time than adaptive algorithms and more than a fixed algorithm. Table 2 represents the comparison operation between the routing 
methods with and without using the GA tool for the used topology. Besides, the use of the sorting principle has been tested with different routing algorithms, and the results of this comparison showed that the result of the sorting algorithm is better than a random algorithm (without sorting) for different routing algorithms, and especially when it used with large topology.

Table (2): A Comparison operation between the routing methods with and without using GA tool for the used topology.

\begin{tabular}{ccc}
\hline \multicolumn{3}{c}{ Accepted Rate \% } \\
\hline Routing method & $\begin{array}{c}\text { Without using GA } \\
\text { tool }\end{array}$ & With using GA tool \\
\hline Fixed & 99.95 & 100 \\
\hline Alternate & 99.68 & 100 \\
\hline Adaptive & 98.18 & 98.21 \\
\hline
\end{tabular}

\section{CONCLUSIONS}

In this paper, the GA has been utilized for the routing methods to solve the RWA issue in AON by simulating traffic with the RWA methods on all-optical WDM systems. By comparing the three routing methods, and essentially with large networks, the rate of the adaptive method was higher than alternate and fixed methods, but it takes longer process time and less resource usage. Therefore, this method is the most convenient among the others and preferred to utilize for large systems. The increment in the number of wavelengths yields increasing in the percentage of requests approval essentially at the beginning, while around the saturation level it is clear that is unworthy to add more wavelengths. Increasing the number of the wavelength affects significantly on the dynamic methods to improve the approval rate with minimal wavelength number. By utilizing GA tool, there was an amelioration for the rate of acceptance requests concerning applying the routing method without using GA tool with the same number of wavelengths, and Implementation of the Genetic Algorithm by using Matlab language very easy because there is GA tool, but the process of implementation is slow essentially with high iterations. There are many suggestions to use the proposed method, its additional overhead, and its effect on the network traffic. Since this work used the GA algorithm over network nodes without wavelength conversions, so the same genetic algorithm model proposed here can be used to solve the RWA problem for networks in both cases with and without wavelength mohammed.saeed@dpu.edu.krd; firas.alfaqe@ nawroz.edu.krd conversion in their nodes. Another suggestion is to work on reducing the computation time of the proposed genetic algorithm since the current simulations run the GA procedure for each connection request.

\section{REFERENCES}

Sivanandam S.N, and Deepa S.N., (2008). Introduction to Genetic Algorithm. ISBN: 9783-540-73189 4. Springer-Verlag Berlin Heidelberg. DOI: 10.1007/978-3-540-73190-0, https://link.springer.com/book/10.1007/978-3$\underline{540-73190-0}$

Lea D. P., (2004). "Soft Computing Approaches to Routing and Wavelength - Routed Optical Network. Ph.D. Thesis, North Carolina State University. ISBN: 978-0-496-96134-4, https://dl.acm.org/doi/book/10.5555/1087600

Chattenjee B.Ch. Sarma N., Pratim P., (2013). Review and Performance Analysis on Routing and Wavelength Assignment Approaches for Optical Networks, IETE Technical Review, 30:1, 12-23. DOI: 10.4103/0256-4602.107335, https://www.tandfonline.com/doi/abs/10.4103/ $\underline{0256-4602.107335}$

Melanie M., (1999). An Introduction to Genetic Algorithms. A Bradford Book The MIT Press Cambridge, Massachusetts, London, England. Fifth printing. ISBN 0-262-13316-4 (HB), 0-262-63185-7 (PB).

Rashed A., Kavian Y.S., Ansari K., and Chassemlooy Z., (2011). Dynamic Routing and Wavelength Assignment: Artificial Bee Colony Optimization. $13^{\text {th }}$ ICTON. DOI: 10.1109/ICTON.2011.5971015, https://ieeexplore.ieee.org/document/5971015

Ozdaglar A.E., and Bertsekas D.P., (2003). Routing And Wavelength Assignment in optical Network. IEEE/ACM Transactions on Networking, Vol. 11, Issue 2. DOI: 10.1109/TNET.2003.810321,

https://ieeexplore.ieee.org/document/1194822

Wason A., and Kaler R.S., (2011). Wavelength assignment algorithms for WDM optical networks. Optik -International Journal for Light and Electron Optics 122(2011):877-880. DOI: $10.1016 /$ j.ijleo.2010.06.013

Jue J.P. (2001). Lightpath Establishment in Wavelength-Routed WDM Optical Networks. In: Ruan L., Du DZ. (eds) Optical Networks. Network Theory and Applications, Vol. 6. Springer, Boston, MA. DOI: 10.1007/978-1-4613-0291-9_5, https://link.springer.com/chapter/10.1007\%2F 978-1-4613-0291-9_5

Chen, L. (2005). Dynamic resource allocation in WDM networks with optical bypass and waveband switching. Ph.D. Thesis. 
Department of Electrical Engineering and Computer Science, Massachusetts Institute of Technology. https://dspace.mit.edu/handle/1721.1/34021

Mokhtar A., and Azizoglu M., (1998). Adaptive Wavelength Routing in All-optical Network. IEEE/ACM Transactions on Networking, Vol. 6, Issue 2. pp: 197-206. DOI: 10.1109/90.664268.

https://ieeexplore.ieee.org/document/664268

Hsieh T., (2003). Dynamic WDM network performance: The impact of banding in reconfigurable optical add/drop multiplexers. M.Sc. Thesis in Electrical Engineering, University of Toronto.

Glenstrup, A. J. (2002). Optimised Design and Analysis of All-Optical Networks. Ph.D. Thesis, Technical University of Denmark, DK-2800.

https://backend.orbit.dtu.dk/ws/files/3018067/ Glenstrup-PhDThesis-OptimisedDesign.pdf

Kaya, Y., Uyar, M., and Tekin, R. (2011). A Novel Crossover Operator for Genetic Algorithms: Ring Crossover. ArXiv, abs/1105.0355. https://arxiv.org/abs/1105.0355

Yussof S., Aziz R., See O. H. , Abdul Ghapar A. , and Md Din M., (2009). A coarse-grained parallel genetic algorithm with migration for shortest path routing problem. HPCC '09. 11th IEEE International Conference on, pp. 615-621. DOI: 10.1109/HPCC.2009.25, https://ieeexplore.ieee.org/document/5167053

Saeed M. G., (2013). "Performance Improvement In Computer Networks By Using Intelligent Algorithm To Solve RWA Problem, MSc. Thesis of science in computer science, 2013, Zakho university, Duhok, Iraq.

Rouskas G.N., Perros H.G. (2002) A Tutorial on Optical Networks. In: Gregori E., Anastasi G., Basagni S. (eds) Advanced Lectures on Networking. NETWORKING 2002. Lecture Notes in Computer Science, vol 2497. Springer, Berlin, Heidelberg. doi: 10.1007/3-540-36162-6_7,

https://link.springer.com/chapter/10.1007/3-54 0-36162-6_7

Mustafa F. M., and Al-Jumailly, Tariq A., (2017). Buffer less All-Optical WDM Networks with Dynamic Traffic, Academic Journal of Nawroz University (AJNU), Vol.6(1), No.(10), Pages:13-25.

DOI:

10.25007/issn.2520-789X.

https://journals.nawroz.edu.krd/index.php/ajnu /index

Chu, X., Li, B., and Chlamtac, I. (2003). Wavelength converter placement under different RWA algorithms in wavelength-routed all-optical networks. IEEE Trans. Communications, 51, 607-617.

DOI:10.1109/TCOMM.2003.810834,

https://ieeexplore.ieee.org/document/1199286

Fenger, C., (2004). Performance Evaluations for Dynamic Wavelength Routed All-Optical Multifiber Networks. Telecommunication Systems 25, 117-127, https://link.springer.com/article/10.1023/B:TE LS.0000011199.88148.91

Lechowicz P., and Walkowiak K., (2016). Genetic algorithm for routing and spectrum allocation in elastic optical networks. Conference, IEEE, Wroclaw, Poland. DOI: 10.1109/ENIC.2016.047.

https://ieeexplore.ieee.org/document/7838076

Monoyios et al., (2016). Indirect crosstalk-aware routing and wavelength assignment in transparent optical networks with the use of Genetic Algorithms, in the 18th (ICTON) Conference, Trento, Italy. Electronic ISSN: 2161-2064.

DOI: 10.1109/ICTON.2016.7550355.

https://ieeexplore.ieee.org/document/7550355/ authors\#authors

Hsu Ch., Cho H., and Fang Sh., (2017). Solving routing and wavelength assignment problem with maximum edge-disjoint paths. Journal of Industrial \& Management Optimization, 13 (2): 1065 - 1084. DOI: 10.3934/jimo.2016062. https://mail.aimsciences.org/article/doi/10.393 4/jimo.2016062

Batista C., Teixeira D., Coelho Th., and Araújo J., (2018). Static-Traffic Routing and Wavelength Assignment in Transparent WDM Networks Using Genetic Algorithm. IFIP Latin American Networking Conference - IFIP LANC 2018 Session 2: Network Management - ISBN: 978-1-4503-5922-1 São Paulo, Brazil. DOI: 10.1145/3277103.3277126.

https://dl.acm.org/doi/abs/10.1145/3277103.32 77126

Garcia A. R., López L. R., Basile F. R. M., (2018). Meta Heuristic for WDM Optical Networks without Wavelength Conversion. Journal of Telecommunication, Electronic and Computer Engineering (JTEC), Vol. 10, No. 1-5, pp 127-131. E-ISSN: 2289-8131. https://journal.utem.edu.my/index.php/jtec/arti cle/view/3643

Kaur H., Rattan M., (2019). Improved offline multi-objective routing and wavelength assignment in optical networks. Front. Optoelectron.12, 433-444. DOI: 10.1007/s12200-019-0850-4.

https://link.springer.com/article/10.1007\%2Fs 12200-019-0850-4 